\title{
The effect of subcutaneous local spraying of Pseudomonas aeruginosa preparation to reduce postoperative drainage time in patients with breast cancer
}

\author{
Quanyi Long ${ }^{1}$, Jia Zhang ${ }^{1}$, Bin Wei ${ }^{2}$, Jiahao $\mathrm{Qi}^{3}$, Hongjiang $\mathrm{Li}^{1}$ \\ ${ }^{1}$ Department of Breast Surgery, West China Hospital, Sichuan University, Chengdu, China; ${ }^{2}$ West China School of Public Health, Sichuan \\ University, West China Campus of Sichuan University, Chengdu, China; ${ }^{3}$ Department of Epidemiology, School of Public Health, Shanxi Medical \\ University, Taiyuan, China \\ Contributions: (I) Conception and design: Q Long, H Li; (II) Administrative support: None; (III) Provision of study materials or patients: None; (IV) \\ Collection and assembly of data: Q Long, J Zhang; (V) Data analysis and interpretation: J Zhang, B Wei, J Qi; (VI) Manuscript writing: All authors; \\ (VII) Final approval of manuscript: All authors. \\ Correspondence to: Hongjiang Li. Department of breast surgery, West China Hospital, Sichuan University, No.37 Guoxue Alley, Wuhou District, \\ Chengdu, China. Email: lihongiiang@sohu.com.
}

\begin{abstract}
Background: To evaluate the efficacy of subcutaneous local spraying of Pseudomonas aeruginosa (P. aeruginosa) preparation (PAP) to reduce the postoperative drainage time in patients with breast cancer (BC).

Methods: The PAP was prepared from an inactivated P. aeruginosa-mannose sensitive hemagglutinin (PAMSHA) strain, a genetically engineered heat-inactivated PA strain with, mannose-sensitive binding activity, which can induce tumor cell apoptosis. This study is its retrospective nature, a total of 65 BC patients (PAP group) who underwent subcutaneous local spraying of PAP in the operation area at West China Hospital of Sichuan University between June 2019 and October 2019, and 32 BC patients without treatment (non-PAP group, without intraoperative subcutaneous local spraying of PAP in the operation area) were enrolled. Daily drainage volume, drainage time, presence of seroma after extubation, and treatment complications (such as fever, incision infection, and flap necrosis) were recorded.
\end{abstract}

Results: No significant differences in age, height, weight, age of initial menstruation, menopause, body mass index (BMI), tumor size, lymph node metastasis, or treatment complications were found between the two groups $(\mathrm{P}>0.05)$. Drainage volume and drainage time in the PAP group were significantly lower than those in the non-PAP group $(\mathrm{P}<0.001)$. Additionally, the incidence of seroma after extubation was significantly lower in the PAP group compared to the non-PAP group $(\mathrm{P}=0.009)$.

Conclusions: Subcutaneous local spraying of PAP on the operation area may be helpful to reduce drainage volume, shorten drainage time, and prevent the occurrence of seroma after BC surgery.

Keywords: Breast cancer (BC); drainage time; Pseudomonas aeruginosa preparation (P. aeruginosa preparation); subcutaneous local spraying

Submitted Sep 27, 2020. Accepted for publication Dec 16, 2020.

doi: $10.21037 / g s-20-797$

View this article at: http://dx.doi.org/10.21037/gs-20-797

\section{Introduction}

Breast cancer (BC) is the most frequent cancer in women of childbearing age and has the highest incidence and mortality rates among all female malignant tumors. Currently, about 2.89 million new cases of female BC arise globally each year, accounting for $24.2 \%$ of all female malignant tumors (1). According to a report published by the National Cancer Center of China in 2019, the incidence of BC in China was about 304,000 cases per year in 2015, with higher incidences in developed regions (2). Surgery 


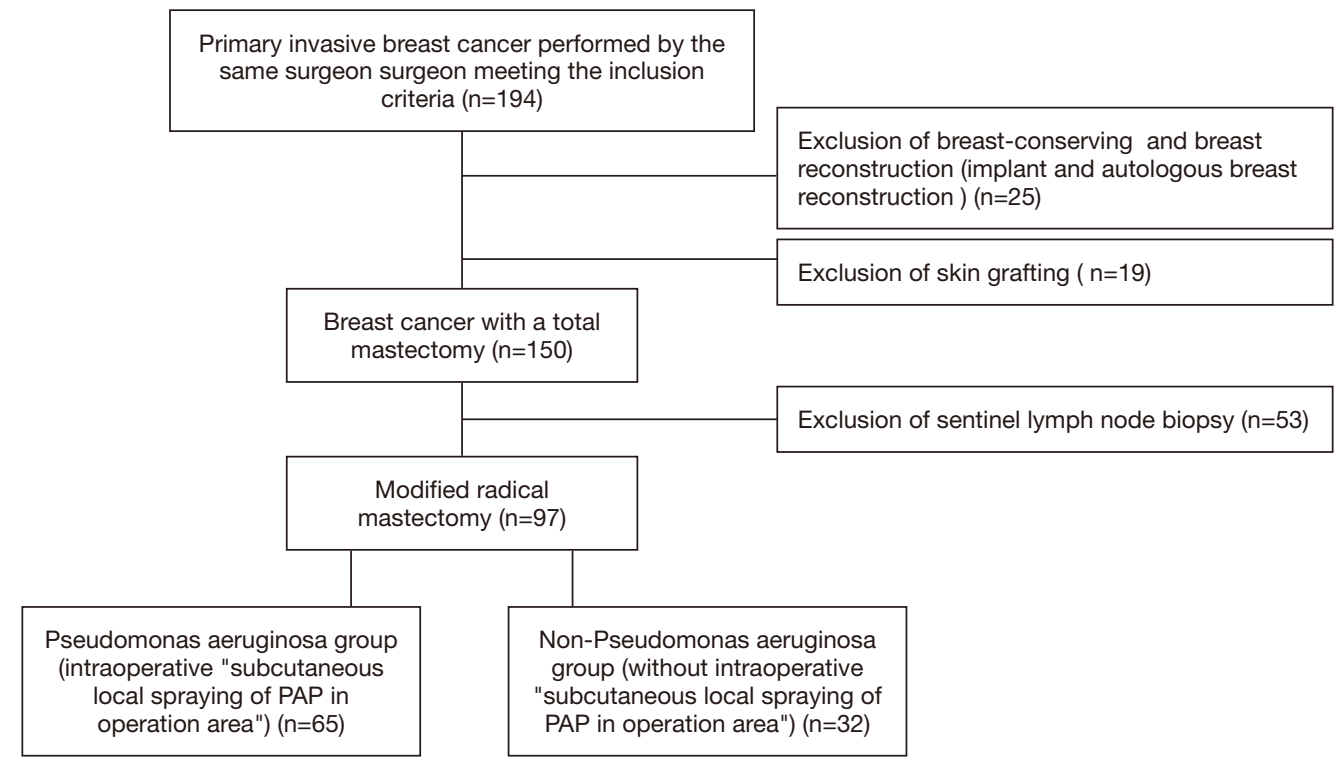

Figure 1 Screening process of study subjects.

remains the best treatment approach for BC, followed by chemotherapy, radiotherapy, endocrine therapy, targeted therapy, immunotherapy, and gene therapy (3). Yet, surgery is often affected by multiple factors, such as wide surgical scope, large surgical wounds, bleeding, and exudation which result in large amounts of postoperative drainage and are difficult resolve in a short time frame. These complications often delay the time of the next treatment (such as radiotherapy and chemotherapy) and increase social and medical costs. This study reviewed the treatments of 65 cases of $\mathrm{BC}$ by subcutaneous local spraying of Pseudomonas aeruginosa (PAP) on the operation area at West China Hospital of Sichuan University between June 2019 and October 2019. We determined that this specific treatment significantly reduced postoperative drainage volume and shortened drainage time.

We present the following article in accordance with the STROBE reporting checklist (available at http://dx.doi. org/10.21037/gs-20-797).

\section{Methods}

The study was approved by the ethics committee of the West China Hospital of Sichuan University. Written informed consent was obtained prior to the study. All procedures performed in this study involving human participants were in accordance with the Declaration of Helsinki (as revised in 2013).

\section{Clinical information}

BC patients whose surgeries were performed by the same surgeon from June 2019 to October 2019 at West China Hospital of Sichuan University were included in this study. Inclusion criteria were the following: (I) female patients; (II) exclusion of distant metastasis of BC with Chest CT, abdominal CT, gynecological color Doppler ultrasound, and bone scans; (III) pathologically confirmed with primary invasive BC; (IV) patients with no past BC surgery; (V) patients with $\mathrm{BC}$ unrelated to pregnancy. Exclusion criteria were as follows: (I) patients undergoing breast-conserving surgery and breast reconstruction (implant and autologous breast reconstruction); (II) patients undergoing sentinel lymph node biopsy (including internal mammary sentinel lymph node biopsy); (III) patients undergoing skin grafting in the operation area.

A total of 97 patients with modified radical mastectomy for $\mathrm{BC}$ were selected the inclusion and exclusion criteria. Sixty-five patients, aged 32-81 years, with an average age of $51.94 \pm 10.777$ years, were intraoperatively assigned to the $P$. aeruginosa treatment group (PAP group); meanwhile, 32 patients, aged 33-74 years, with an average age of $53.97 \pm 11.488$ years, were assigned to the non- $P$. aeruginosa group (non-PAP group). Postoperatively, there was one silicone drainage tube for parasternal and axillary draining for each patient. The screening process of the subjects is shown in Figure 1. 


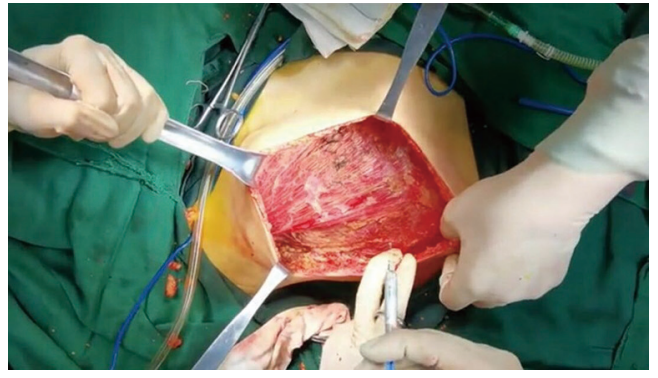

Figure 2 Subcutaneous local spraying of P. aeruginosa preparation.

\section{Data collection}

Basic data of patients and relevant information on surgery were preoperatively collected. The occurrence of postoperative complications, such as fever, infection, skin flap necrosis, and seroma within 1 month after extubation; and cumulative total drainage volume and drainage time (time after removal of both drainage tubes) were also collected. Wound infection and skin flap necrosis were analyzed when changing the wound dressing (every 3 days) by the same two wound therapists.

Extubation criteria included the following: drainage volume of the single tube for 2 consecutive days was $\leq 10 \mathrm{~mL} /$ day, and the drainage tube was smooth and without seroma. Seroma was defined according to the Marquez (4) and Srivastava (5) criteria, with seroma being considered any subcutaneous or axillary effusion that requires clinical or radiographic guidance for aspiration after mastectomy and axillary dissection.

\section{Grouping}

In the PAP group, PAP $(2 \mathrm{~mL})$ was sprayed on the wounds in operation area before placement of drainage tube (Figure 2), and was followed by placing two drainage tubes in the same area (a single drainage tube was placed in the sternum and in the axilla, respectively). Then, the tube was clamped, and an incision was made with an interrupted fullthickness suture. After closing the incision, the drainage tube clamp was opened, and finally, external low negative pressure sustained suction was initiated.

In the non-PAP group, complete hemostasis was performed on the surgical wound, with direct placement of two drainage tubes (a single drainage tube was placed in the sternum and in the axilla, respectively). The incision was made with an interrupted full-thickness suture. After

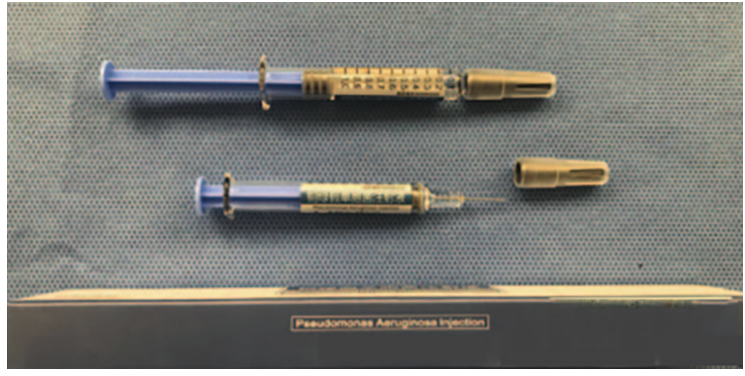

Figure 3 Pseudomonas aeruginosa preparation injection and its outer packaging.

closing the incision, external low negative pressure sustained suction was initiated.

\section{$P A P$}

The PAP, manufactured by Beijing Wanter Biopharmaceutical Company (Figure 3), was prepared from an inactivated $\mathrm{P}$. aeruginosa-mannose sensitive hemagglutinin (PA-MSHA) strain, a genetically engineered heatinactivated PA strain with mannose-sensitive binding activity, which can induce tumor cell apoptosis.

\section{Statistical analysis}

Statistical analysis was performed by SPSS (version 17.0) software using a two-sided probability test. A $\mathrm{P}$ value $<0.05$ was considered as statistically significant. Counting data are expressed as cases or percentages, and measurement data are expressed as mean and standard deviation (mean $\pm \mathrm{SD}$ ). Any differences between the two groups were analyzed by $t$-test, Mann-Whitney $\mathrm{U}$ test, or chi-square test.

\section{Results}

\section{Comparison in baseline information}

A total of 97 cases were included (65 in the PAP group and 32 in the non-PAP group). No significant differences in age, height, weight, age of initial menstruation, menopause, body mass index (BMI), tumor size, or lymph node metastasis were found between the two groups $(\mathrm{P}>0.05$; Table 1).

\section{Comparison in efficacy}

Total drainage volume and drainage time in the PAP 
Table 1 Basic information of patients

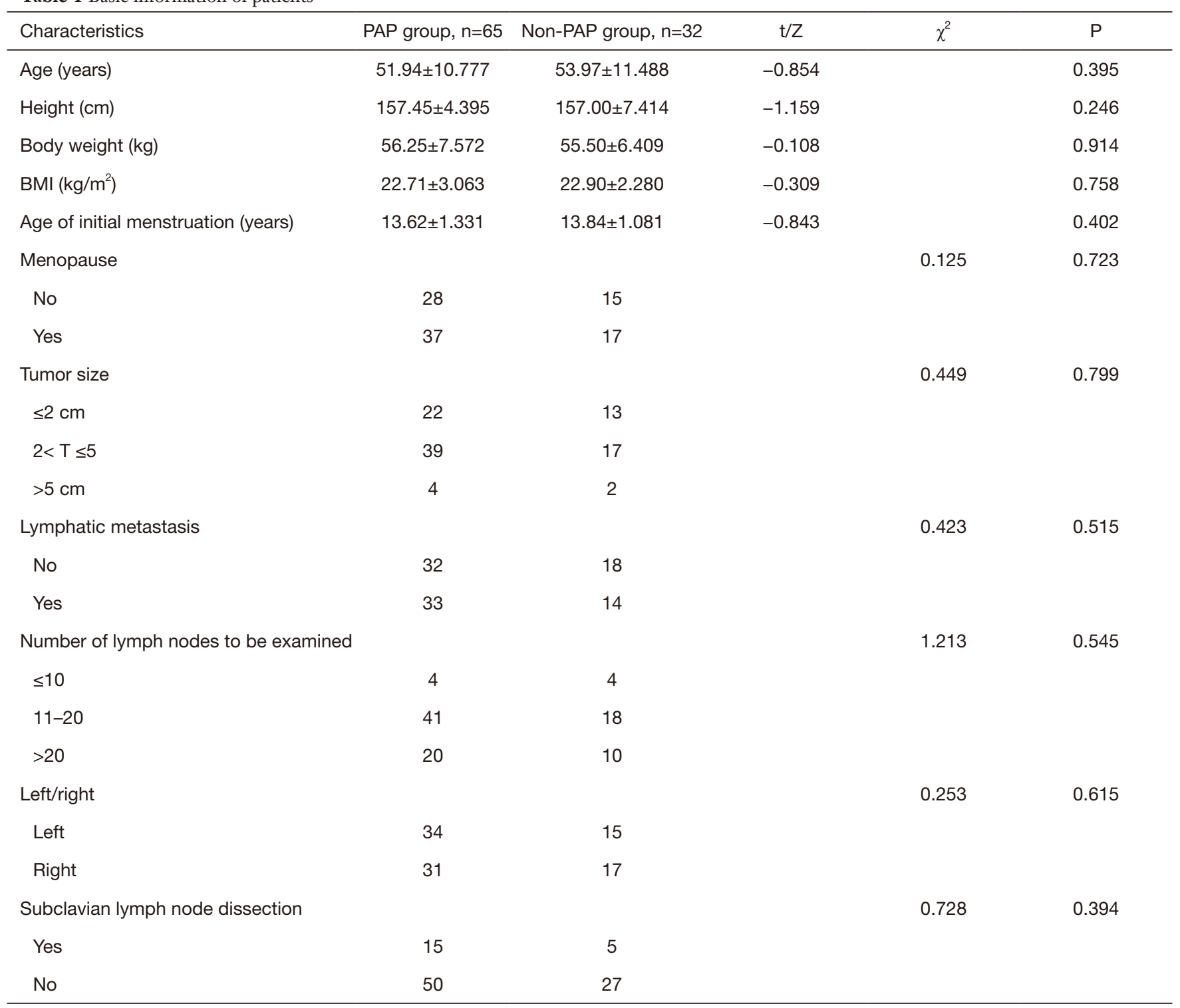

PAP, Pseudomonas aeruginosa preparation.

group were both significantly lower compared to those in the non-PAP group (median volume $526.93 \pm 273.009$ vs. $908.23 \pm 594.072 \mathrm{~mL}$, median days $14.14 \pm 4.934 v s$. $20.03 \pm 8.837 ; \mathrm{P}<0.001$ ) (Table 2). The incidence of seroma after extubation in the PAP group was significantly lower than that in the non-PAP group $\left(\chi^{2}=6.908, \mathrm{P}=0.009\right)$ (Table 3).

\section{Comparison in complications}

Fever wound infections and skin flap necrosis, occurred in both groups without a statistical difference $(\mathrm{P}>0.05)$ (Table 4). Six cases of fever (below $39{ }^{\circ} \mathrm{C}$ ), which spontaneously disappeared within 24 hours, were observed in the PAP group.

\section{Discussion}

Surgery has an essential and irreplaceable role in the comprehensive treatment of BC. Although sentinel lymph node biopsy can help some patients avoid axillary lymph node dissection, modified radical mastectomy remains the 
Table 2 Total drainage volume and drainage time in the PAP and non-PAP groups

\begin{tabular}{|c|c|c|c|c|}
\hline Characteristics & PAP group, $n=65$ & Non-PAP group, $n=32$ & $t / z$ & $P$ \\
\hline Drainage time (days) & $14.14 \pm 4.934$ & $20.03 \pm 8.837$ & -3.645 & $<0.001$ \\
\hline
\end{tabular}

PAP, Pseudomonas aeruginosa preparation.

Table 3 The incidence of seroma after extubation in the PAP and non-PAP groups

\begin{tabular}{lcccc}
\hline Characteristic & PAP group, $n=65$ & Non-PAP group, $n=32$ & t/z & $P$ \\
\hline Incidence of seroma after extubation (cases) & 3 & 7 & 6.908 & 0.009 \\
\hline
\end{tabular}

PAP, Pseudomonas aeruginosa preparation.

Table 4 Complications after treatment in the PAP and non-PAP groups

\begin{tabular}{|c|c|c|c|c|}
\hline Characteristics & PAP group, $n=65$ & Non-PAP group, $\mathrm{n}=32$ & $t / z$ & $\mathrm{P}$ \\
\hline Wound infection (cases) & 1 & 2 & 1.588 & 0.208 \\
\hline Skin flap necrosis (cases) & 1 & 1 & 0.267 & 0.605 \\
\hline
\end{tabular}

PAP, Pseudomonas aeruginosa preparation.

primary surgical approach for BC in developing countries. Due to the wide scope of operation, large surgical wounds and extensive use of electrotome intraoperatively may cause considerably more damage to adipose tissue, blood and lymphatic vessels, which may, in turn, lead to increased postoperative exudates, higher drainage volume, longer drainage time, and even increased risk of infection and seroma.

Thus far, several chemical and physical methods, such as various types of negative pressure drainage, suture sewing, ultrasonic knife (6), affected side upperlimb immobilization, intracavitary injection of talc $(7,8)$, local injection of protein glue (9), and streptococcal preparation OK-432 (10) have been tested with the aim of reducing postoperative drainage volume and drainage time, and preventing the occurrence of seroma. Yet, every method has some limitations. For example, talc causes pain and subcutaneous induration and other adverse reactions, while intraoperative sutures and OK-432 may increase operation time (11). It is also unclear whether long-term upper limb immobilization may increase the risk of exercise limitation.

In the present study, we evaluated the efficacy of subcutaneous local spraying of PAP to reduce the postoperative drainage time in patients with $\mathrm{BC}$. The
PAP was prepared from an inactivated PA-MSHA strain, a genetically engineered heat-inactivated PA strain with mannos-sensitive binding activity, which can induce tumor cell apoptosis $(12,13)$. This genetically engineered strain can promote activation of human peripheral blood mononuclear cells, induce action of helper $\mathrm{T}$ cells and $B$ cells, enhance the activity of natural killer cells, and increase levels of multiple cytokines (14). PAP has been used for the treatment of malignant pleural and pericardial effusions (15) and intractable chylous fistula in neck surgery (16). However, few studies have evaluated its effect on drainage time in BC patients undergoing modified radical mastectomy. Our data showed that the drainage volume was significantly reduced in the PAP group compared with the non-PAP group $(526.93 \pm 273.009$ vs. $908.23 \pm 594.072 \mathrm{~mL}, \mathrm{P}<0.001)$, and the drainage time was significantly shortened $(14.14 \pm 4.934$ vs. $20.03 \pm 8.837$ days, $\mathrm{P}<0.001)$. We propose that subcutaneous local spraying of PAP can help trigger tissue repair response, cause a strong local sterile inflammatory reaction between the tissue surfaces, and promote the skin and wounds in the operation area to be more prone to adhesion and fibrosis. Thus, PAP can reduce the total drainage volume after BC surgery and drainage time.

Srivastava et al. (5) have suggested that seroma is a side 
effect rather than a complication after BC surgery. In a report on wound healing and postoperative complications of BC surgery, Chiappa et al. (17) reported that the incidence of seroma was $37.5 \%$ in the conventional electrosurgical group. In this study, the incidence after extubation in the PAP group was not only lower than that in the non-PAP group (4.615 vs. 21.875, $\mathrm{P}=0.009$ ), but was also lower than those reported in the above literature. This data suggests that subcutaneous local spraying of PAP on the operation area can reduce the incidence of seroma in $\mathrm{BC}$ patients undergoing surgery.

The standard for extubation in this study was a drainage that was less than or equal to $10 \mathrm{~mL}$ for 2 consecutive days before removal of the drainage tube. At present, there is no report on drainage time after BC surgery with the above standard extubation. In addition, studies reporting on the extubation index and drainage time are not consistent. In their study, Lohani et al. (18) removed the drainage tube when the drainage was less than or equal to $20 \mathrm{~mL}$ for 2 consecutive days; the drainage time was 11 days without intervention, and the incidence of seroma was $32.6 \%$. Gogna et al. (19) reported that the drainage tube was removed when the drainage was less than $30 \mathrm{~mL}$ for 1 day; the drainage time was 13.7 days without intervention, and the incidence of seroma was $44 \%$. Moreover, Chiappa et al. (17) reported that the drainage tube was removed when the daily drainage volume decreased to less than 50 $\mathrm{ml}$. While the average drainage time in the control group was $10.93 \pm 5.173$ days, there are some reported cases of the drainage tube being removed when the daily drainage volume was reduced to less than $100 \mathrm{~mL}$ (20). Nevertheless, the extubation criteria in this study was stricter, which is why the drainage time was shorter in our PAP group, but longer than that reported in the other above-mentioned studies. Lower incidence of seroma in this study may also be related to the strict extubation criteria.

Because PAP is an inactivated bacterial preparation, fever is the most common adverse reaction after injection. As expected, our results showed that the number of cases with fever was higher in the PAP group than in the non-PAP group (six cases $v s$. three cases, $\mathrm{P}=0.623$ ). In the $\mathrm{PAP}$ group, fever (lower than $39^{\circ} \mathrm{C}$ ) lasted for $6-12$ hours and was spontaneously alleviated within 24 hours postoperatively, with no increase in the incidence of other complications being observed.

The main limitation of this study is its retrospective nature. Future long-term, large-sample size randomized controlled trials are necessary to further verify our findings.

\section{Conclusions}

Subcutaneous local spraying of PAP on the operation area may be used to reduce postoperative drainage volume and shorten drainage time, and may reduce the formation of postoperative seroma. This treatment could alleviate local symptoms of patients, improve the patients' quality of life, and save valuable time for further treatment.

\section{Acknowledgments}

Funding: None.

\section{Footnote}

Reporting Checklist: The authors have completed the STROBE reporting checklist. Available at http://dx.doi. org/10.21037/gs-20-797

Data Sharing Statement: Available at http://dx.doi. org/10.21037/gs-20-797

Conflicts of Interest: All authors have completed the ICMJE uniform disclosure form (available at http://dx.doi. org/10.21037/gs-20-797). The authors have no conflicts of interest to declare.

Ethical Statement: The authors are accountable for all aspects of the work in ensuring that questions related to the accuracy or integrity of any part of the work are appropriately investigated and resolved. The study was approved by the ethics committee of the West China Hospital of Sichuan University. Written informed consent was obtained prior to the study. All procedures performed in this study involving human participants were in accordance with the Declaration of Helsinki (as revised in 2013).

Open Access Statement: This is an Open Access article distributed in accordance with the Creative Commons Attribution-NonCommercial-NoDerivs 4.0 International License (CC BY-NC-ND 4.0), which permits the noncommercial replication and distribution of the article with the strict proviso that no changes or edits are made and the original work is properly cited (including links to both the formal publication through the relevant DOI and the license). See: https://creativecommons.org/licenses/by-nc-nd/4.0/. 


\section{References}

1. Bray F, Ferlay J, Soerjomataram I, et al. Global cancer statistics 2018: GLOBOCAN estimates of incidence and mortality worldwide for 36 cancers in 185 countries. CA Cancer J Clin 2018;68:394-424.

2. Zhang Q, Xiao Q, Guo R, et al. Applications of rib sparing technique in internal mammary vessels exposure of abdominal free flap breast reconstructions: a 12year single-center experience of 215 cases. Gland Surg 2019;8:477-85.

3. Liu J, Liu JL. Breast cancer treatment decisions: from individualized treatment to precision medicine. Journal of Clinical Medical Literature (Electronic Edition). 2016;3:1579-9.

4. Marquez JE, Kapadia K, Ghosh K, et al. Efficacy of Fibrin Sealants in Preventing Seroma Formation in Reduction Mammaplasty: A Single Surgeon's Experience. Ann Plast Surg 2020;85:S41-3.

5. Srivastava V, Basu S, Shukla VK. Seroma formation after breast cancer surgery: what we have learned in the last two decades. J Breast Cancer 2012;15:373-80.

6. Iovino F, Gatta G, Auriemma PP, et al. Late Shoulder-Arm Morbidity Using Ultrasound Scalpel in Axillary Dissection for Breast Cancer: A Retrospective Analysis. J Surg Res 2019;233:88-95.

7. Luo K, Yang HS, YL D. Treatment of 34 cases of lymphatic leakage after operation of breast cancer with talc powder suspension. Journal of Guiyang Medical College 2010;35:431-2.

8. Yu YZ, Hu XC, Zhao H, et al. Clinical study on treatment of refractory subcutaneous effusion of breast cancer with talc powder suspension after operation. Journal of Clinical Surgery 2013;6:485.

9. Qiu ZL, Wang Y, Y. F. Application of Ankejiao in modified radical operation of breast cancer. Journal of Practical Diagnosis and Therapy 2006;20:676-7.

10. Yang Y, Gao E, Liu X, et al. Effectiveness of OK-432 (Sapylin) to reduce seroma formation after axillary lymphadenectomy for breast cancer. Ann Surg Oncol 2013;20:1500-4.

11. Kong D, Liu Y, Li Z, et al. OK-432 (Sapylin) Reduces Seroma Formation After Axillary Lymphadenectomy in Breast Cancer. J Invest Surg 2017;30:1-5.

12. Zhang C, Zhang Z, Wang L, et al. Pseudomonas aeruginosa-mannose sensitive hemagglutinin injection treated cytokine-induced killer cells combined with chemotherapy in the treatment of malignancies. Int Immunopharmacol 2017;51:57-65.

13. Li T, Dong ZR, Guo ZY, et al. Mannose-mediated inhibitory effects of PA-MSHA on invasion and metastasis of hepatocellular carcinoma via EGFR/Akt/lkappaBbeta/ NF-kappaB pathway. Liver Int 2015;35:1416-29.

14. Li T, Yang L, Fu SJ, et al. Subcutaneous Injections of the Mannose-Sensitive Hemagglutination Pilus Strain of Pseudomonas aeruginosa Stimulate Host Immunity, Reduce Bladder Cancer Size and Improve Tumor Survival in Mice. Cell Biochem Biophys 2015;73:245-52.

15. Zhu JQ, Wu XM. Evaluation of Pseudomonas aeruginosa injection therapy on malignant pleural effusion and pericardial effusion. Chin J Clin Oncol Rehabil 2010;2:93-5.

16. Wei T, Liu F, Li Z, et al. Novel Management of Intractable Cervical Chylous Fistula with Local Application of Pseudomonas aeruginosa Injection. Otolaryngol Head Neck Surg 2015;153:561-5.

17. Chiappa C, Fachinetti A, Boeri C, et al. Wound healing and postsurgical complications in breast cancer surgery: a comparison between PEAK PlasmaBlade and conventional electrosurgery - a preliminary report of a case series. Ann Surg Treat Res 2018;95:129-34.

18. Lohani KR, Kumar C, Kataria K, et al. Role of tranexamic acid in axillary lymph node dissection in breast cancer patients. Breast J 2020;26:1316-20.

19. Gogna S, Goyal P. Prospective randomized study on effect of tranexamic acid on wound drainage following modified radical mastectomy for cancer breast. Int J Curr Res 2015;7:16192-4.

20. Abass MO, Gismalla MDA, Alsheikh AA, et al. Axillary Lymph Node Dissection for Breast Cancer: Efficacy and Complication in Developing Countries. J Glob Oncol 2018;4:1-8.

(English Language Editors: L. Gray and J. Gray)

Cite this article as: Long Q, Zhang J, Wei B, Qi J, Li H. The effect of subcutaneous local spraying of Pseudomonas aeruginosa preparation to reduce postoperative drainage time in patients with breast cancer. Gland Surg 2020;9(6):2064-2070. doi: $10.21037 / g s-20-797$ 